\title{
A VISÃo dA PEdiatria ACERCA dA GRAVIdez NA AdolescÊNCIA: UM ESTUDO BIBLIOCRAFICO
}

\author{
Romeu Gomes ${ }^{1}$ \\ Eliane M.G.O.Fonseca ${ }^{2}$ \\ Álvaro J.M.O.Veiga ${ }^{3}$
}

Gomes R, Fonseca EMGO, Veiga AJMO. A visão da pediatria acerca da gravidez na adolescência: um estudo bibliográfico. Rev Latino-am Enfermagem 2002 maio-junho; 10(3):408-14.

O artigo objetiva analisar o conteúdo da produção bibliográfica, em três periódicos nacionais de Pediatria, no período de 1990 a 1999, acerca da gravidez na adolescência, identificando as explicações para a ocorrência desse tipo de gravidez e encaminhamentos sugeridos para a temática. As variáveis do estudo (periódico; ano de publicação; formação e atuação dos autores; palavras-chave; idéias associadas ao tema e metodologia utilizada) foram investigadas através de freqüências e cruzamentos. Na abordagem qualitativa, procurou-se analisar as explicações e as recomendações da Pediatria sobre o assunto. Dos 1437 artigos, apenas 1,1\% relacionavam-se ao tema. Controvérsias sobre o predomínio do risco biológico foram observadas nos artigos. Dentre as conclusões, aponta-se a necessidade da perspectiva interdisciplinar na abordagem do tema, considerando a complexidade dos aspectos que envolvem a gravidez na adolescência.

DESCRITORES: gravidez, adolescência, pediatria

\section{A PEDIATRIC VIEW ON PREGNANCY AMONG ADOLESCENTS: A BIBLIOGRAPHICAL STUDY}

This study analyzes the content of the scientific production of three national pediatric journals from 1990 to 1999, concerning pregnancy among adolescents and identifies the justifications for this occurrence and the follow-up suggested. The following variables were investigated according to their frequency: journal; year of publication; authors background; keywords; ideas associated with the subject in question; methodology. A qualitative approach enabled an analysis of the pediatric explanations and recommendations concerning this theme. Among 1437 papers, only 1.1\% were related to the subject. Controversies regarding the importance of the biological risk involving the adolescents' lives were observed in the papers. In sum, the authors point out the need for an interdisciplinary perspective in approaching this theme, considering the complexity of the aspects involved in the pregnancy among adolescents.

DESCRIPTORS: pregnancy, adolescence, pediatrics

\footnotetext{
${ }_{1}^{1}$ Doutor em Saúde Pública, Pesquisador do Instituto Fernandes Figueira/FIOCRUZ, e-mail: romeu@iff.fiocruz.br; ${ }^{2}$ Pediatra, Professor de Pediatria da Universidade Gama Filho e Faculdade de Medicina Souza Marques, Doutoranda do Instituto Fernandes Figueira/FIOCRUZ; ${ }^{3}$ Pediatra, Professor de Pediatria da Faculdade de Medicina de Petrópolis. Doutorando do Instituto Fernandes Figueira/FIOCRUZ
} 


\section{LA VISIÓN dE LA PEDIATRÍA ACERCA DEL EMBARAZO EN LA ADOLESCENCIA: UN ESTUDIO BIBLIOGRAFICO}

El estudio analiza el contenido de la producción bibliográfica, en las tres revistas nacionales de Pediatría, desde 1990 hasta 1998, con respecto al embarazo en la adolescencia. A través de las frecuencias y cruzamientos de las variables del estudio (revista; año de publicación; formación y actuación de los autores; términos claves; asociación de ideas al tema y metodología utilizada) fue hecho el escenario da la producción. En termos cualitativos se intento hacer una análisis de las explicaciones y las recomendaciones de la Pediatría con respecto al tema. De los 1437 artículos, solamente $1.1 \%$ tuvieron relación con el tema. La controversia al respecto de la importancia del riesgo biológico que involucra en la vida del adolescente era evidente en los artículos. Entre las conclusiones, se enfatiza la necesidad de la perspectiva interdisciplinaria al abordar el tema, considerando los aspectos tan complejos que envuelven el embarazo en la adolescencia.

DESCRIPTORES: embarazo, adolescencia, pediatría

\section{INTRODUÇÃO}

A gestação na adolescência representa um desafio para os profissionais de saúde, educadores, governo e sociedade em geral, podendo acarretar conseqüências sociais, emocionais e físicas, que se entrelaçam num todo indissociável ${ }^{(1-2)}$.

No Brasil, sua incidência vem aumentando. A pesquisa Nacional de Demografia e Saúde, de 1996, demonstrou que, embora a taxa de fecundidade tenha baixado no Brasil, nos últimos anos, a fecundidade da população adolescente parece estar aumentando: $18 \%$ das adolescentes de 15 a 19 anos já tiveram, pelo menos, uma gravidez ${ }^{(3)}$.

A gravidez precoce tem sido associada à maior morbimortalidade materna, prematuridade e baixo peso ao nascer. Esses dados são controversos, e diversos autores assinalam que essas conseqüências são mais freqüentes no grupo de 10 a 14 anos e associam-se com precária assistência pré-natal e ao parto ${ }^{(3-8)}$. Há, também, outros problemas que são potencializados pelas privações socioeconômicas prévias: menor chance de completar a escolaridade, reduzindo as oportunidades de emprego; tendência maior à construção de famílias numerosas e de separações; maior chance de os filhos adoecerem, sofrerem acidentes e de apresentarem baixo rendimento escolar ${ }^{(3,6)}$.

O pediatra encontra-se numa posição favorável para colaborar na inversão desse problema, devido ao seu contato diário com préadolescentes, adolescentes e pais, atuando na prevenção da gestação não desejada e do aborto sem segurança, propiciando o acesso à contracepção. E, uma vez ocorrida a gravidez, ele deve atuar junto às adolescentes grávidas e seus filhos, à família da adolescente e ao pai da criança, garantindo condições satisfatórias de assistência ${ }^{(4-5,9)}$.

Assim, compreender como esse campo da Medicina trata o assunto em questão é de fundamental importância para que os profissionais de saúde em geral possam, a partir do posicionamento dessa área, participar de uma discussão mais ampla, visando a uma atuação multiprofissional frente à gravidez na adolescente.

A partir dessas considerações, este artigo objetiva analisar o conteúdo da produção bibliográfica, em três periódicos nacionais de 
Pediatria, no período de 1990 a 1999, acerca da gravidez na adolescência, identificando as explicações para a ocorrência desse tipo de gravidez e encaminhamentos sugeridos para a questão.

\section{METODOLOGIA}

Foram estudados três periódicos nacionais representativos na área, no período de 1990 a 1999: Arquivo Brasileiro de Pediatria (Periódico 1); Jornal de Pediatria (Periódico 2) e Pediatria Moderna (Periódico 3). Em relação ao periódico 1 , foram considerados os volumes publicados no período de 1994 a 1997, uma vez que essa publicação tornou-se periódico a partir de 1994 (antes era um suplemento do Jornal Brasileiro de Medicina) e teve sua publicação interrompida no ano de 1997 . Foram identificados 16 artigos que possuíam, em seu título ou em suas palavras-chave, as expressões gravidez ou gestação na adolescência $^{(2,10-24)}$. A análise do material fundamentou-se na articulação das abordagens quantitativa e qualitativa, uma vez que essas duas perspectivas, de naturezas diferentes, são complementares na busca de melhor se aproximar de uma realidade que se quer conhecer $^{(25)}$.

$\mathrm{Na}$ análise relativa à metodologia, foi utilizada a seguinte classificação: (I) Ensaio (estudo que se baseia unicamente na experiência do autor); (II) Estudo de caso clínico (estudo de casuística); (III) Estudo descritivo (estudo que não envolve metodologia epidemiológica, embora trabalhe os dados quantitativamente); (IV) Estudo epidemiológico (estudo de distribuição de determinado fenômeno de saúde/doença, em determinado local, espaço e grupo populacional); (V) Pesquisa qualitativa (estudo de crenças, valores, percepções, representações e sentidos atribuídos); (VI) Revisão bibliográfica (estudo baseado em consulta bibliográfica); (VII) Estudo quanti-qualitativo (estudo que articula as duas abordagens); (VIII) Estudo baseado em grupo psicopedagógico ou em oficina de trabalho.

Em termos quantitativos, foram investigadas as seguintes variáveis: periódico; ano de publicação; formação e atuação dos autores; palavras chaves; idéias associadas ao tema; metodologia utilizada. Essas variáveis foram analisadas nos programas Dbase III Plus e Epi-Info 6.0, por meio de freqüências e cruzamentos.

Em termos qualitativos, a partir de adaptações $^{(26)}$, procurou-se analisar os modelos explicativos da Pediatria sobre o assunto, por meio das seguintes questões: (a) Como a Pediatria explica a ocorrência da gravidez na adolescência? (b) Quais são os encaminhamentos sugeridos para se lidar com a gravidez na adolescência?

$\mathrm{Na}$ análise qualitativa, foi excluído um artigo $^{(24)}$ por não discutir a temática do estudo, mas apenas mencioná-la.

O procedimento de análise das respostas a essas questões encontradas nos artigos estudados seguiu os seguintes passos: (a) identificação das idéias centrais; (b) comparação entre as diferentes idéias presentes nos textos; (c) descoberta de eixos em torno dos quais giravam os argumentos dos autores acerca das questões investigadas e (d) elaboração de um discurso crítico a partir dos posicionamentos dos autores dos artigos estudados. 


\section{RESULTADOS DO ESTUDO}

Como pode ser observado na Tabela 1 , verificou-se a existência de poucos artigos relacionados ao objeto da pesquisa, totalizando 16 e representando $1,1 \%$ do conjunto da produção. O Periódico 3, em termos absolutos, apresentou 10 artigos, concentrando $62,5 \%$ do total de artigos relacionados ao estudo. Já, em termos relativos, levando-se em consideração número de artigos publicados e número de artigos relacionados ao tema estudado, é o Periódico 1 que concentra o maior percentual de produção (2,2\%). Observa-se, também, que o ano de 1992 concentra 4 artigos, correspondendo a $25 \%$ dos artigos estudados, numa seqüência irregular.

Tabela 1 - Distribuição de artigos por periódico e ano de produção, 1990-1999*

\begin{tabular}{|c|c|c|c|c|c|c|c|c|}
\hline \multirow[t]{2}{*}{ Ano } & \multicolumn{4}{|c|}{ Artigos Publicados } & \multicolumn{4}{|c|}{ Artigos relacionados ao estudo } \\
\hline & $\begin{array}{c}\text { Perió dico } \\
1\end{array}$ & $\begin{array}{c}\text { Periódico } \\
2\end{array}$ & $\begin{array}{c}\text { Periódico } \\
3\end{array}$ & Total & $\begin{array}{c}\text { Periódico } \\
1\end{array}$ & $\begin{array}{c}\text { Periódico } \\
2\end{array}$ & $\begin{array}{c}\text { Periódico } \\
3\end{array}$ & Total \\
\hline 1990 & - & 44 & 29 & 73 & - & 0 & 0 & 0 \\
\hline 1991 & - & 67 & 75 & 159 & - & 0 & 0 & 0 \\
\hline 1992 & - & 58 & 78 & 146 & - & 1 & 3 & 4 \\
\hline 1993 & - & 48 & 102 & 177 & - & 0 & 0 & 0 \\
\hline 1994 & 19 & 39 & 115 & 173 & 1 & 1 & 1 & 3 \\
\hline 1995 & 20 & 48 & 76 & 144 & 0 & 1 & 1 & 2 \\
\hline 1996 & 30 & 63 & 89 & 182 & 1 & 0 & 2 & 3 \\
\hline 1997 & 21 & 67 & 116 & 204 & 0 & 0 & 2 & 2 \\
\hline 1998 & 0 & 79 & 98 & 177 & 0 & 1 & 1 & 2 \\
\hline 1999 & 0 & 21 & 35 & 56 & 0 & 0 & 0 & 0 \\
\hline Total & 90 & 534 & 813 & 1437 & 2 & 4 & 10 & 16 \\
\hline
\end{tabular}

* Observação: no ano de 1999 está considerado apenas o $1^{\circ}$ semestre

Em termos de formação e/ou atuação do principal autor, ressaltou-se a concentração de pediatras $(56,3 \%)$, seguidos por psicólogos (25\%), psicanalistas $(6,3 \%)$ e sociólogo $(6,3 \%)$. No que se refere a profissionais da área de saúde mental, estes publicaram no periódico 3, que possui um espaço destinado à articulação entre psicologia e pediatria, concentrando $60 \%$ dos artigos.

Em relação à metodologia utilizada pelos autores, conforme Tabela 2, destacaram-se o estudo descritivo e o estudo baseado em discussão de uma oficina de trabalho ou de grupo, ambos com 5 artigos, representando cada um $31,3 \%$ no conjunto de métodos empregados. Em geral, observou-se a existência de apenas 2 artigos baseados em estudos epidemiológicos, representando 12,5\% dos métodos utilizados. Constatou-se, também, a ausência de pesquisas qualitativas. Cruzando periódico com metodologia empregada, verificou-se a utilização de estudo descritivo nos três periódicos. No Periódico 3 , houve maior diversidade de métodos, com uma concentração em estudo baseados em discussão de uma oficina de trabalho ou de grupos. 
Tabela 2 - Distribuição dos artigos por método e periódico

\begin{tabular}{lcccc}
\hline \multicolumn{1}{c}{ Método } & $\begin{array}{c}\text { Periódico } \\
1\end{array}$ & $\begin{array}{c}\text { Periódico } \\
2\end{array}$ & $\begin{array}{c}\text { Periódico } \\
3\end{array}$ & Total \\
\hline Ensaio & 0 & 0 & 1 & 1 \\
Estudo de caso & 0 & 0 & 0 & 0 \\
Descritivo & 2 & 1 & 2 & 5 \\
Epidemiológico & 0 & 1 & 1 & 2 \\
Qualitativo & 0 & 0 & 0 & 0 \\
Bibliográfico & 0 & 0 & 2 & 2 \\
Quanti-qualitivo & 0 & 1 & 0 & 1 \\
Oficina & 0 & 0 & 5 & 5 \\
$\quad$ Total & 2 & 3 & 11 & 16 \\
\hline
\end{tabular}

No que se refere a aspectos médicos voltados para a gravidez na adolescência - tais como morbimortalidade materna, prematuridade e baixo peso ao nascer constatou-se que apenas 5 artigos $^{(2,10-11,14-15)}$, no conjunto de 16 , trouxeram dados sobre tais riscos, embora apenas $1^{(10)}$ tenha se baseado em amostra numericamente significativa.

Em relação aos dados qualitativos, quanto à ocorrência da gravidez na adolescência, verificou-se que esta foi explicada pelos autores pela comprovação da capacidade de gerar um filho, atestando, assim, a feminilidade da adolescente $e^{(16-17,19-22)}$. A falta de informações acerca da contracepção e da sexualidade em geral também contribuiu para a ocorrência da gravidez na adolescência. Isso foi evidenciado tanto pela ausência de orientação no âmbito familiar, como de programas educativos mais amplos ${ }^{(2,12-13,16-17)}$. Outra explicação para o fato de uma adolescente engravidar situou-se no plano psicológico, por meio de um processo de idealização, que busca, com a gestação, suprir ou resolver carências de ordens afetiva, física e socioeconômica ${ }^{(12,16)}$. O início precoce da vida sexual do adolescente associado à impulsividade, própria dessa faixa etária, também contribuiu, segundo alguns autores, para a ocorrência da temática em questão ${ }^{(13,17,23)}$.

Em relação aos encaminhamentos sugeridos para se lidar com a gravidez na adolescência, observou-se que nem todos os artigos estudados trabalharam sugestões nesse sentido. Do conjunto, 10 fizeram encaminhamentos. No entanto, destes últimos, apenas 3 artigos qualificaram suas propostas $^{(10,12,20)}$. Os demais emitiram considerações bastante genéricas, tais como promover a atenção ao adolescente, à sua família e ao companheiro; desenvolver programas de educação sexual e estabelecer uma maior compreensão da adolescência.

Dentre as recomendações para se lidar com a gravidez na adolescência, uma idéia que perpassou mais de um autor foi da necessidade de se reconhecer a existência da atividade sexual nessa faixa etária para promover a prevenção ${ }^{(10,12)}$. Outra sugestão foi a capacitação dos profissionais de saúde para melhor atuar junto à temática em questão ${ }^{(20)}$.

\section{DISCUSSÃO}

O estudo revelou, no âmbito dos artigos analisados, a quase inexistência de dados nacionais acerca da incidência da gravidez na adolescência e de suas repercussões, apoiados em investigações epidemiológicas. Por outro lado, a ausência de estudos qualitativos acerca da temática também foi observada. Isso caminha, de forma oposta, a uma tendência atual de se estudarem os fenômenos relacionados aos contextos pessoais e sociais no campo da saúde, por meio dessa abordagem $^{(1)}$. 
A participação do Periódico 3 , com artigos relacionados ao tema, contribui de forma decisiva, como visto, para o número total de estudos publicados. Esses estudos, em geral, foram de autoria de profissionais de saúde mental e basearam-se em discussões de oficinas de trabalho ou de grupos. Essa característica explica-se pelo fato de esse periódico conter uma seção de Psicologia e Pediatria.

Em relação à formação do primeiro autor, destaca-se o fato de existir apenas um profissional do campo da sociologia, revelando a ausência de uma maior discussão social que - tema envolve e apontando para a necessidade de uma maior integração entre as ciências sociais e clínica médica ${ }^{(1)}$.

No que se refere a riscos biológicos da gravidez na adolescência, observou-se que esses, em geral, foram citados, mas não tiveram seu estudo aprofundado. Ressaltou-se, ainda, que tais artigos não contextualizaram esses riscos, tomando como referências as condições socioeconômicas e culturais, conforme vem sendo recomendado ${ }^{(7-8)}$.

Constatou-se a ausência da figura masculina na discussão da temática. $O$ que contraria a recomendação de se incluir o parceiro e pai da criança nos programas sobre gravidez e paternidade na adolescência ${ }^{(4)}$.

\section{REFERÊNCIAS BIBLIOGRÁFICAS}

1. Organización Panamericana de la Salud, Organización Mundial de la Salud, Fondo de Población de las Naciones Unidas. El embarazo en la adolescencia: lineamentos para el diseño de investigaciones de aspectos socioculturales. México (DF): Organización Panamericana de la Salud; 1996.

2. Abdallah VOS, Mayrink L, Gonçalves RMP, Nishioka SA. Gravidez na adolescência: experiência de um hospital universitário. Pediatria Moderna 1998 setembro; 34(9):561-70.
Em termos de recomendações, verificou-se que as propostas sobre a temática eram genéricas, revelando pouco amadurecimento do assunto no âmbito da Pediatria. Não havia recomendações de normas de prevenção e de assistência à temática estudada. Essas normas vêm sendo recentemente publicadas no âmbito da Pediatria $^{(4)}$.

\section{CONCLUSÕES}

Com base na revisão realizada, concluiuse que os conteúdos dos artigos reforçam a necessidade de se abordar a temática a partir de uma perspectiva interdisciplinar. A gravidez na adolescência envolve, dente outros aspectos, num campo mais amplo, questões relacionadas ao ser adolescente e, num sentido mais específico, aspectos ligados à sexualidade. Diante dessa complexidade, a discussão não pode se restringir à clínica pediátrica, mas, sim, ampliar o debate para além do campo biomédico, tentando compreender como o processo de gravidez é vivido na adolescência, com suas múltiplas implicações, no sentido de se buscarem medidas de prevenção frente à temática em questão.

3. Camarano AA. Fecundidade e anticoncepção da população de 15 a 19 anos. Anais do Seminário Gravidez na Adolescência do Projeto de Estudos da Mulher no Brasil; 1998 julho; 35-46.

4. Academia Americana de Pediatria. Tendências da gravidez na adolescência: qual o rumo, quando e porque? Pediatrics [edição brasileira] 1999 abril; 3(3):175-7.

5. Academia Americana de Pediatria. Gravidez na Adolescência: Tendências e Questões Atuais: 1998. Pediatrics [edição brasileira] 1999 junho; 3(6):439-44.

6. Bruno ZV, Bailey PE. Gravidez em adolescentes no Ceará: maternidade ou aborto. Anais do Seminário Gravidez na Adolescência do Projeto de Estudos da Mulher no Brasil 1998 julho; 57-66. 
7. Stern C. El embarazo en la adolescencia como problema público: una visión crítica. Salud Pública de México 1997 marzo-abril; 39 (2):137-43.

8. Stern C. Significado e implicaciones para distintos sectores sociales. Demos - Carta Demográfica sobre México 1995; 8:11-2.

9. Nações Unidas. Resumo do Programa de Ação da Conferência Internacional sobre População e Desenvolvimento: 5-13 setembro 1994. Cairo (Egito): Nações Unidas; 1995.

10. Bhering CA, Bhering JA, Mendonça EG. Estudo descritivo e comparativo de adolescentes grávidas e seus conceptos no município de Barra Mansa - RJ. Arq Bras Pediat 1994 maio-junho; 1(2):47-52.

11. Sá DSS, Silva MFPR, Magalhães MAS, Epifânio Netto A, Rego TMS. O perfil da mãe adolescente e do seu filho no Hospital Maternidade Carmela Dutra - Rio de Janeiro. Arq Bras Pediat 1996 setembro-outubro; 3(5):139-42.

12. Taquete SR. Sexo e gravidez na adolescência. J Pediatr 1992 março-abril; 68(3/4)135-9.

13. Costa COM, Pinho JFC, Martins SJ. Aspectos psicossociais e sexuais de gestantes adolescentes em Belém-Pará. J Pediatr 1995 maio-junho; 71(3):151-7.

14. Mariotoni GG, Barros AAB Filho. A gravidez na adolescência é fator de risco para o baixo peso ao nascer? J Pediatr 1998 março-abril; 74(2):107-13.

15. Espiridião S, Júlio AG, Marcolim M, Silva AN, Oliverio IAP, Nascimento JP, et al. Avaliação dos recém-nascidos de mães adolescentes no Hospital Municipal de Santo André. Correlação com algumas variáveis maternas. Pediatr Mod 1992 dezembro; 28(7):526-9.

16. Marques SMB, Chalem E, Ângelo MLB. Gravidez na adolescência. Pediatr Mod 1992 dezembro; 28(7):58492.

17. Higuchi ALC, Souza AM, Neves MN, Augusto CA, Costa MC, Mattar G. Experiência de um trabalho educativo em grupo com adolescentes do Hospital do Servidor Público Estadual. Pediatr Mod 1994 abril; 30(2):131-42.

18. Schiller R. Gravidez na adolescência: uma questão de hereditariedade? Pediatr Mod 1994 outubro; 30(6):9848.

19. Schiller ROM, Kornives DC, Faria MF, Guazzelli C, Mattar R, Silveira MLM. Gravidez na adolescência: grupos psicopedagógicos. Pediatr Mod 1995 dezembro; 31(7):1132-5.

20. Cavasin S, Arruda S. Gravidez na adolescência: um outro enfoque. Pediatr Mod 1996 fevereiro; 32(1):84-7. 21. Chalem E, Ângelo MLB. A mãe adolescente. Pediatr Mod 1996 abril; 32(2):188-91.

22. Silva MCP. Aspectos psicológicos do aborto em adolescentes. Pediatr Mod 1997 junho; 33(6):458-60.

23. Carneiro $H$, Ladessa LECL, Rodrigues Júnior OM, Vaccari VL, Lerner T. Preocupações sexuais de mulheres adolescentes no Brasil. Pediatr Mod 1997 agosto; 33(8):645-53.
24. Pusch CM, Pusch MC. Grupos pedagógicos de adolescentes. Pediatr Mod 1992 dezembro; 27(7):554-7. 25. Minayo MCS, Sanches O. Quantitativo-qualitativo: oposição ou complementaridade? Cad Saúde Pública 1993 julho; 9(3):239-62.

26. Alves PC. A experiência da enfermidade: considerações teóricas. Cad Saúde Pública 1993 julho; 9(3):263-71. 